\title{
EDITORIAL
}

\section{What is the Important Issue to Prevent the Postoperative Crohn's Disease?}

\author{
You Sun Kim \\ Department of Internal Medicine, Seoul Paik Hospital, Inje University College of Medicine, Seoul, Korea
}

\section{Article: Clinical and Endoscopic Recurrence after Surgical Resection in Patients with Crohn's Disease (Intest Res 2014;12:117-123)}

Crohn's disease (CD) is a chronic inflammatory disease that affects the entire gastrointestinal tract. Complications caused by CD, including strictures, perforations, and fistulas, often lead patients to undergo surgery, including intestinal resection. Several studies have reported that $50-80 \%$ of CD patients undergo the surgery during their lifetime. In Korea, Ye et al. reported that the cumulative probability of intestinal resection at 1,5 , and 10-15 years after a diagnosis of $C D$ was $15.5 \%, 25.0 \%$, and $32.8 \%$, respectively. ${ }^{2}$ In a more extensive study by Park et al., the cumulative probability of intestinal resection at 10,20, and 30 years after a diagnosis of CD was $43.5 \%, 70.0 \%$, and $76.1 \%$, respectively. ${ }^{3}$ These data suggest that the intestinal resection rate of Korean CD patients is not lower than that of Western patients and that Korean CD patients may, in fact, have a similar clinical course to Western CD patients.

However, intestinal resection does not cure CD, and many patients suffer from postoperative recurrences of the disease. The recurrence of disease necessitates repeated intestinal resection, with up to $50 \%$ of patients undergoing additional surgery after 20 years. ${ }^{4}$ Ye et al. also reported that $30.8 \%$ of the patients who received an intestinal operation required a repeat operation after 10 years. $^{2}$

Postoperative recurrence can be defined as clinical recur-

Received March 5, 2014. Revised March 10, 2014

Accepted March 12, 2014.

Correspondence to You Sun Kim, Division of Gastroenterology, Department of Internal Medicine, Seoul Paik Hospital, Inje University College of Medicine, 9 Mareunnae-ro, Jung-gu, Seoul 100-032, Korea. Tel: +82-2-2270-0012,

Fax: +82-2-2270-0257,E-mail:yousunk69@korea.com

Financial support: None. Conflict of interest: None. rence or endoscopic recurrence. Postoperative endoscopic recurrence (PER), which is usually quantified using a Rutgeerts scoring system, ${ }^{5}$ typically precedes clinical recurrence and PER was reported to occur in almost $58 \%$ of CD patients within 1 year of intestinal resection. ${ }^{6}$ In this issue, Lee et al. conducted a retrospective study to evaluate the factors influencing and recurrence rates of postoperative $\mathrm{CD}^{7}$ Thirtyfour patients who were regularly followed-up for at least 1 year after intestinal resection were analyzed. The mean follow-up period after intestinal resection was 65.4 months and their results showed a cumulative clinical recurrence rate of $8.8 \%, 12.5 \%$, and $33.5 \%$ at 1,2 , and 4 years, respectively. Among 21 patients who had received colonoscopy after their intestinal resection, the cumulative PER was $42.9 \%$ and $66.1 \%$ at 1 and 2 years, respectively. Therefore, 27 out of 34 patients $(79.1 \%)$ showed clinical or endoscopic recurrence after intestinal resection. Although the sample size in this study was small, and the duration of follow-up was relatively short, these results are comparable with data from Western countries. Given the increased incidence of CD in Korea and the resulting increase in intestinal resection, the prevention and management of postoperative recurrence is an important issue for Korean clinicians. As such, Lee et al's studywhich is the first to report the recurrence rate and risk factors for CD after intestinal resection in Korea-is of particular relevance. $^{7}$

A risk-stratified strategy involving tailored therapy according to high/low risk patient groups in order to prevent postoperative recurrence of $\mathrm{CD}$ was recently proposed. This postoperative management strategy included determining the risk factors of patients, identifying PER by performing

(c) Copyright 2014. Korean Association for the Study of Intestinal Diseases. All rights reserved.

This is an Open Access article distributed under the terms of the Creative Commons Attribution Non-Commercial License (http://creativecommons.org/licenses/by-nc/3.0)

which permits unrestricted non-commercial use, distribution, and reproduction in any medium, provided the original work is properly cited. 
colonoscopy 6-12 months after surgery, and promptly introducing preventive medications in order to reduce the recurrence rate. High-risk factors included smoking, perforating behavior, perianal disease, young age, repetitive surgery, and extensive bowel disease. ${ }^{8}$ In contrast, low-risk factors included the stricture behavior and old age at diagnosis. Until now, 5-aminosalicylates (5-ASA) and probiotics have not been proven to be effective in preventing postoperative recurrence of $\mathrm{CD}$, whilst metronidazole has shown shortterm benefits (3 months). Azathioprine/6-mercaptopurine (AZT/6-MP) has been proven to be effective and combined therapy with metronidazole and AZT/6-MP was more effective than metronidazole alone. In this issue, Lee et al. also demonstrated that patients who took immunomodulators to prevent postoperative recurrence had significantly lower recurrence rates than the 5-ASA group $(P=0.042){ }^{7}$ These results are comparable with the large scale study by Park et al. in which early use of AZT was associated with a significantly delayed requirement for intestinal resection (hazard ratio: $0.63,95 \%$ confidence interval: $0.46-0.85)^{3}{ }^{3}$

Recent studies have also focused on the effectiveness of tumor necrosis factor (TNF)- $\alpha$ antagonists in treating and preventing PER, especially in high-risk patients. TNF- $\alpha$ antagonist therapy (including infliximab and adalimumab) seemed more effective than AZT in preventing and treating PER in many studies. ${ }^{9,10}$ Despite these studies being performed with few patients and without long-term follow-up, and although safety and cost issues should also be considered, the use of TNF- $\alpha$ antagonists is currently recommended as appropriate treatment in the high-risk patient group. In Korea, the efficacy of TNF- $\alpha$ antagonists in preventing the postoperative recurrence of $\mathrm{CD}$ has not yet been reported. Therefore, prospective large-scale studies are needed to clarify the burden of postoperative recurrence of $\mathrm{CD}$ and to determine the high-risk factors of recurrence in Korean CD patients. Furthermore, the efficacy of TNF- $\alpha$ antagonists in improving the long-term prognosis and quality of life of highrisk patients should be further investigated.

\section{REFERENCES}

1. Sandborn WJ, Feagan BG. The efficacy of azathioprine and 6-mercaptopurine for the prevention of postoperative recurrence in patients with Crohn's disease remains uncertain. Gastroenterology 2004;127:990-993.

2. Ye BD, Yang SK, Cho YK, et al. Clinical features and long-term prognosis of Crohn's disease in Korea. Scand J Gastroenterol 2010;45:1178-1185.

3. Park SH, Yang SK, Park SK, et al. Long-term Prognosis of Crohn's Disease and Its Temporal Change Between 1981 and 2012: A Hospital-based Cohort Study from Korea. Inflamm Bowel Dis 2014;20:488-494.

4. Yamamoto T. Factors affecting recurrence after surgery for Crohn's disease. World J Gastroenterol 2005;11:3971-3979.

5. Rutgeerts P, Geboes K, Vantrappen G, Beyls J, Kerremans R, Hiele M. Predictability of the postoperative course of Crohn's disease. Gastroenterology 1990;99:956-963.

6. Pascua M, Su C, Lewis JD, Brensinger C, Lichtenstein GR. Metaanalysis: factors predicting post-operative recurrence with placebo therapy in patients with Crohn's disease. Aliment Pharmacol Ther 2008;28:545-556.

7. Lee YW, Lee KM, Chung WC, Paik CN, Sung HJ, Oh YS. Clinical and endoscopic recurrence after surgical resection in patients with Crohn's disease. Intest Res 2014;12:117-123.

8. El-Hachem S, Regueiro M. Postoperative Crohn's disease: prevention and treatment. Expert Rev Gastroenterol Hepatol 2009;3:249-256.

9. Yoshida K, Fukunaga K, Ikeuchi H, et al. Scheduled infliximab monotherapy to prevent recurrence of Crohn's disease following ileocolic or ileal resection: a 3-year prospective randomized open trial. Inflamm Bowel Dis 2012;18:1617-1623.

10. Kotze PG, Saad-Hossne R. Biological therapy for the prevention and treatment of postoperative endoscopic recurrence in Crohn's disease: Time for acceptance? Intest Res 2013;11:256260. 